\title{
Elección profesional y sesgo de selección: evaluación de los sistemas de admisión universitaria en Chile en un contexto de agenda pro-inclusión
}

\author{
Carlos René Rodríguez Garcés \\ Geraldo Padilla Fuentes
}

\begin{abstract}
Resumen: Bajo la prerrogativa de una mayor inclusión, el sistema de admisión universitaria en Chile ha realizado en las últimas dos décadas una serie de reformas cuyo efecto concita interés evaluativo. Con tal propósito, haciendo uso de bases de datos oficiales, el artículo analiza los perfiles y cambios de tendencia en la educación superior de carácter universitaria-selectiva con ocasión del proceso de reforma. Los resultados manifiestan que, pese a la reconfiguración de los programas y consiguiente aumento de los alumnos que logran ingresar a la educación superior, persisten las desigualdades en las posibilidades de selección profesional con base a atributos de capital social y cultural. Más allá de las capacidades y aptitudes que las reformas buscan poner en el centro de la cuestión, las pruebas de admisión encubren un sesgo estructural de un sistema educativo difícil de cambiar. Sesgo de selección que, si bien se evidencia claramente en el proceso de admisión, alcanza la opción profesional en términos de institución y programa elegido, su permanencia y titulación oportuna.
\end{abstract}

Palabras clave: Selección universitaria. Inclusión social. Sistema de admisión. Sesgo de selección. Educación superior.

\section{Escolha profissional e viés de seleção: avaliação dos sistemas de admissão universitário no Chile no contexto da agenda pró-inclusão}

Resumo: Sob a prerrogativa de uma maior inclusão, o sistema de admissão universitária no Chile realizou nas duas últimas décadas uma série de reformas cujo efeito desperta interesse de avaliação. Com tal propósito, fazendo uso de bases de dados oficiais, o artigo analisa os perfis e mudanças de tendências na educação superior de caráter seletivo-universitário no período do processo de reforma. Os resultados mostram que, apesar da reconfiguração dos programas e consequente aumento dos alunos que conseguem ingressar na educação superior, persistem as desigualdades nas possibilidades de escolha profissional com base em atributos de capital social e cultural. Mais além das capacidades e aptidões que as reformas visam colocar no centro da questão, as provas de admissão encobrem um sistema educativo enviesado em sua estrutura e difícil de mudar. Viés de seleção que, se bem fica claramente evidenciado no processo de admissão, atinge a escolha profissional em termos de instituição e programa escolhido, sua permanência e titulação oferecida.

Palavras-chave: Seleção universitária. Inclusão social. Sistema de admissão. Viés de seleção. Educação superior.

\section{Professional choice and selection bias: evaluation of university admission system in Chile in a pro-inclusion agenda context}

Abstract: Focusing on the prerogative of greater inclusion, the Chilean university admissions system has gone through a series of reforms in the last two decades, the effect of which has aroused evaluative interest. Using official databases, the purpose of this article was to analyze the profiles and changes in higher education trends of a university-selective nature as a result of the reform process. In spite of 
Elección profesional y sesgo de selección: evaluación de los sistemas de admisión universitaria en Chile en un contexto de agenda pro-inclusión

redesigned programs and the ensuing increase in the number of students that enter higher education, results revealed that inequalities persist in the opportunities of professional selection based on social and cultural capital attributes. Beyond the skills and aptitudes that the reforms seek to put at the center of the issue, entrance exams conceal the structural bias of an educational system that is difficult to change. Although it is clearly evident in the admissions process, selection bias affects the professional option in terms of institution and program choice, continuity, and timely graduation.

Key words: University selection. Social inclusion. Admissions system. Selection bias. Higher education.

\section{Introdução}

En lo que a sistema de educación superior de carácter universitario se refiere, Chile presenta un modelo dualizado de admisión. Por un lado, una amplia oferta privada con disímiles criterios para la matrícula de nuevos alumnos. Oferta que convive con la proveniente de universidades tradicionales y públicas reguladas por el Sistema Único de Admisión (SUA), mecanismo de selección encargado de ordenar a los postulantes en razón de un puntaje que otorga mayor objetividad al proceso al presentar estándares conocidos y uniformes. El SUA, en la búsqueda por cumplir con criterios de inclusión social e igualdad en las oportunidades de ingreso y desarrollo, ha implementado desde hace ya dos décadas una serie de reformas en su estructura para revertir los fenómenos de exclusión, segregación y elitización que ha denunciado la investigación educativa en su funcionamiento. En este afán se sustituyó la antigua y criticada Prueba de Aptitud Académica (PAA) por una nueva batería de test. Esta Prueba de Selección Universitaria (PSU), aplicada por primera vez en 2004, estaría en teoría más alineada al currículum de carácter oficial implementado universalmente en la enseñanza secundaria $\mathrm{y}$, por ende, menos condicionada por factores de capital sociocultural del alumnado. A ello se suma, como medida de pro-inclusión, la gratuidad para rendirla por todos los estudiantes recién egresados de la educación secundaria, hecho que universaliza su rendición al eliminar la barrera económica que implicaba el alto costo de la inscripción, en especial para los alumnos más vulnerables.

No obstante la incorporación de la PSU es un avance, persisten dudas respecto de su real eficacia, por cuanto la premisa de la homogeneidad del currículum no contempla las disímiles condiciones socio-culturales y laborales en que los establecimientos educativos deben funcionar. Condiciones de aula que dificultan los niveles de cobertura y aprovechamiento curricular. Investigaciones recientes informan que cerca del $43 \%$ de los establecimientos municipales no dicta el currículum completamente (PÉREZ; ORTIZ; PARRA, 2011), a lo que se suma los desiguales niveles de dominio disciplinar y expectativas docentes (RODRÍGUEZ; CASTILLO, 2014; RODRIGUEZ; CASTILLO; SAAVEDRA, 2015). 
A ambas acciones, creación de la PSU y universalización de la inscripción por medio de la gratuidad, se añade en el año 2013 el Ranking de Notas. Reforma al Sistema Único de Admisión Universitaria (SUA) que otorga una mayor ponderación al desempeño educativo, revalorizando la trayectoria escolar y el esfuerzo desplegado en la educación secundaria por parte del alumno que busca hacerse de un cupo en la universidad. Medida que busca subsanar el sesgo de selección e insuficiencia que presentan las pruebas estandarizadas al momento de determinar el ingreso y permanencia en la educación superior, ello con base a la amplia evidencia reportada (BETTS; MOREL, 1999; CATALÁN; SANTELICES, 2014; CLIFFORDSON; ASKLING，2006; CONTRERAS; GALLEGOS; MENESES, 2009; GEISER; STUDLEY, 2002; GIL; PAREDES; SÁNCHEZ, 2013; MEDINA; AGUIRRE; LUENGO, 2014; MENESES; BLANCO, 2006; MENESES; TORO, 2012; MENESES; PARRA; ZENTENO, 2005).

El último hito en el proceso de selección universitaria lo constituye la reciente incorporación de un conjunto de 8 universidades privadas al SUA de un total de 35 existentes. Esta inserción bajo el estatuto de "adscritas" (LARROCAU, 2014), implica en lo inmediato regular cupos y requerimiento de ingreso de parte de una oferta programática anteriormente escindida del sistema que operaba con heterogéneos criterios de admisión. Situación paradigmática considerando que el sector privado atiende entre el 65\% y $70 \%$ de la matrícula de pregrado sin mayores regulaciones, por lo que esta decisión puede seguir fines más académicos que hagan frente a los altos niveles de deserción en sus programas (LEYTON; VÁSQUEZ; FUENZALIDA, 2012).

En el funcionamiento del SUA aguarda la convicción de que, si bien no es posible determinar con certeza el nivel de éxito de los alumnos en la educación superior, la PSU capta determinadas capacidades o conocimientos mínimos que permiten prever la adaptación del estudiante a las exigencias que supone la preparación universitaria (GIL, 2006). Al operar dentro de márgenes de error aceptable, este supuesto merece algunos matices. Al considerar variables sociales ligadas al proceso de selección, en especial los disímiles niveles de renta, inequidad distributiva y acceso a servicios por parte de las diferentes capas sociales, se observan diferenciales niveles educativos entre los estudiantes, tanto en calidad como en pertinencia del conocimiento adquirido. A grosso modo, existiría un sector privado ligado a la excelencia académica y de altos costos económicos, y un sector público o subvencionado ligado a la preparación regular/insuficiente de costos moderados. Este preámbulo hace muchas veces de la universidad un espacio de reproducción de la segregación socioeconómica y académica existente en los niveles básico y secundario, lo que condiciona fuertemente las 
Elección profesional y sesgo de selección: evaluación de los sistemas de admisión universitaria en Chile en un contexto de agenda pro-inclusión

posibilidades de los estudiantes de los sectores más vulnerables de ingresar, permanecer y graduarse de la educación superior (BELLEI, 2013; OCDE, 2009; GONZÁLEZ; URIBE; GONZÁLEZ, 2005). El tipo de habitus institucional, concepto clave en la investigación educativa actual que hace referencia a la relación existente entre la clase social de procedencia y el contexto socio-institucional que acoje a los nuevos estudiantes, genera grados de sentido de pertenencia, compromiso educativo y adquisición de capital social-simbólico particulares (LEYTON; VÁSQUEZ; FUENZALIDA, 2012). De modo que no son solo los estudiantes quienes con base a un determinado perfil académico realizan una elección profesional, sino que también las mismas universidades, amparadas en dicho habitus, seleccionan a sus alumnos. El estatus social de que gozan determinadas instituciones y programas, trae aparejado una serie de cualidades que, pudiendo ayudar a mejorar las condiciones de competencia por ingresar a la educación superior, la mayoría de las veces se elitiza o sobredimensiona con base a características "extra-académicas" (CLIFFORDSON; ASKLING, 2006).

En relación a lo académico, existe en la transición de ciclo un desajuste entre los niveles de exigencia universitarios y las habilidades académicas desarrolladas. Esta experiencia de ajuste relativo se obstaculiza con ocasión de la baja preparación o adecuación académica del estudiante a ritmos de trabajo intenso, descuido del locus interno o desatención de técnicas de estudio grupales e individuales. Esta situación estresante, una vez superada la barrera de selección, afecta parceladamente a los universitarios noveles, situándose mayoritariamente entre los estudiantes provenientes de estratos socioeconómicos bajos, egresados de establecimientos públicos, primera generación y dotados de escaso capital cultural, situación que impacta negativamente en sus niveles de permanencia y éxito académico (CANALES; DE LOS RÍOS, 2009; LEYTON; VÁSQUEZ; FUENZALIDA, 2012). La mala preparación académica de los alumnos de sectores segregados hace necesario dictar cursos curriculares específicos dentro de las carreras para nivelar las falencias de contenido, aunque los recursos y tiempo que conlleva dicha tarea dependen de cada institución (SANZ, 2005).

Si bien los niveles de participación en la educación superior de carácter universitario por parte de estudiantes de estratos socieconómicos bajos han aumentado respecto a décadas anteriores, no se puede obviar el riesgo que poseen de salir de la misma sin concluir sus carreras, el cual es mucho mayor del que tiene el resto de estudiantes. Sobreponerse a handicaps de tipo académico, familiar y social, como ausencia de hábitos de estudio, de contenidos básicos, soportes económicos auxiliares o redes fuertes de apoyo institucional 
(CANALES; DE LOS RÍOS, 2009), es lo que podemos denominar la "seleccción universitaria oculta". Situación que constituye un daño emergente, máxime cuando la inversión económica se realizó mediante créditos contraídos con entidades bancarias, los cuales inobjetablemente deberá pagar. Así entendido, este estudiante desertor se enfrenta al peor de los mundos: contrae una deuda de egresado universitario con las credenciales educativas de un estudiante de enseñanza secundaria (RODRÍGUEZ; CASTILLO, 2015). En la medida que el nivel socioconómico, la dependencia del establecimiento de egreso y el capital educativo-cultural de los padres inciden poderosamente en los niveles de acceso, elección profesional y permanencia del alumnado, se demanda, por parte de las instituciones educativas, un conjunto de medidas anexas al proceso de admisión, las cuales permitan visualizar el rol de las universidades como actores responsables y comprometidos, junto al Estado, de cumplir con criterios de equidad e igualdad de oportunidades.

En consecuencia, más allá de la confiabilidad que pueda tener el SUA y con ocasión del interés que concita la igualdad de oportunidades en el acceso a la educación superior de carácter universitario, resulta de interés indagar en las características de los estudiantes que, logrando superar la barrera de una selección específica, se distribuyen al interior de una oferta de programas e instituciones de atributos muy variados. Se analizan, del contingente de seleccionados en los procesos de admisión universitaria, las elecciones profesionales efectuadas con base al perfil socioeducativo del alumnado y niveles de selectividad de la oferta educativa, haciendo especial hicanpié en la incidencia que tienen las reformas estructurales del SUA en su contribución a la mixturización social de los espacios universitarios. Ello sustentado en la premisa que, bajo coordenadas de reproducción social, las elecciones profesionales realizadas por los estudiantes vulnerables obedecen más a un ajuste de expectativas a la baja que a una genuina opción vocacional.

\section{Metodología}

Muestra

Las unidades de análisis fueron los estudiantes seleccionados en los procesos de admisión a las universidades chilenas y regidas bajo el Sistema Único de Admisión. La información deriva de bases de datos secundarias que provee el Departamento de Evaluación, Medición y Registro Educacional (DEMRE), organismo técnico responsable de la administración del proceso de selección y admisión a la educación superior en Chile. Se trata de datos poblacionales que, en razón a la universalidad alcanzada por la PSU en el presente, 
Elección profesional y sesgo de selección: evaluación de los sistemas de admisión universitaria en Chile en un contexto de agenda pro-inclusión

comprenden para el año 2014 un patrón muestral de 278.623 postulantes, en su mayoría recientemente egresados de enseñanza secundaria.

\section{Diseño}

Con las bases de los procesos de admisión se realiza un análisis estadístico inferencial de tipo descriptivo-correlacional, a fin de configurar los niveles de segmentación en las elecciones profesionales efectuadas con base a atributos socioeducativos del alumnado e institucionales. A nivel de oferta universitaria se construye un indicador que permite ordenar según nivel de selectividad de los programas profesionales. Selectividad establecida con base a la proporción de alumnos que, con determinado puntaje en las PSU de Matemática y Lenguaje, resultaron seleccionados en un determinado programa. Así, usando como referencia el proceso de admisión 2011 se logra generar tres tipologías:

- Programas de Alta selectividad: al menos el 50\% del alumnado seleccionado registra puntajes sobre 650 puntos PSU,

- Programas de Media selectividad: más del 50\% del alumnado seleccionado con rangos de puntaje entre 550 y 650 puntos PSU,

- Programas de Baja selectividad: más del 50\% de su alumnado seleccionado obtuvo puntajes bajo los 550 puntos PSU.

Por otra parte, el análisis de la incidencia que tendrían las medidas de pro-inclusión contempladas en los procesos de reforma al SUA, son efectuadas también controlando el eventual sesgo de agregación. A fin de hacer las bases de datos comparables, se utiliza una muestra ajustada que contempla únicamente la oferta programática existente en los distintos periodos. Modelo que complementa el análisis general realizado.

\section{Resultados y Discusiones}

A partir del año 2007 y mediante un conjunto de acciones afirmativas pro inclusión, el proceso de admisión universitaria revierte la paulatina y constante caída en el número de estudiantes que, habiendo egresado de la enseñanza media o secundaria, rendían los test estandarizados para ocupar algunas de las vacantes ofrecidas por el sistema universitario de carácter selectivo. En el año 2015 alrededor de 280 mil estudiantes se inscribieron para rendir las pruebas de admisión, cifra que representa a la casi totalidad de los egresados en dicho año, haciendo de la participación en el proceso de admisión universitaria un acto de tránsito casi obligatorio y de carácter universal. Si bien en términos formales se evidencia una amplia 
cobertura en la inscripción de los alumnos recientemente egresados, dados los incentivos que supone la gratuidad, en la actualidad tienden a aumentar las proporciones de postulantes autoexcluidos, es decir, estudiantes que habiendo inscrito las pruebas de selección no las rinden. Así mismo, las medidas implementadas pro inclusión no logran revertir el sesgo de selección por atributos socioeducativos del alumnado. Con base a su gran convocatoria e inscripción casi universal, los procesos de admisión congregan en única instancia un variado contingente de participantes de singulares características y atributos cuyos resultados o niveles de logro alcanzados, contrario a lo heterogéneo de su composición, están fuertemente condicionados por factores estructurales, entre ellos la procedencia socioeconómica. Si el talento se distribuye homogéneamente en las diferentes capas sociales y el currículum sobre el cual se alinean los contenidos evaluados por las pruebas de admisión son universales, sería inusual observar desproporcionadas concentraciones de estudiantes de un determinado sector socioeconómico en particulares niveles de rendimiento PSU, como las que se constatan históricamente.

Desde el año 2000 al 2015 una proporción que transita entre un 25\% y 35\% de quienes se inscriben en los procesos de admisión resulta seleccionada para ocupar algunas de las plazas ofrecidas por las instituciones que participan del SUA, cifras que darían cuenta de adecuados estándares de discriminalidad de los procesos de admisión, manteniendo un aceptable equilibrio entre cobertura y selectividad. No obstante ello, los datos también informan de la profunda brecha existente en los niveles de admisión según segmento socioeconómico de pertenencia.

Gráfico 1 - Tasas de selectividad histórica según nivel socioeconómico del postulante

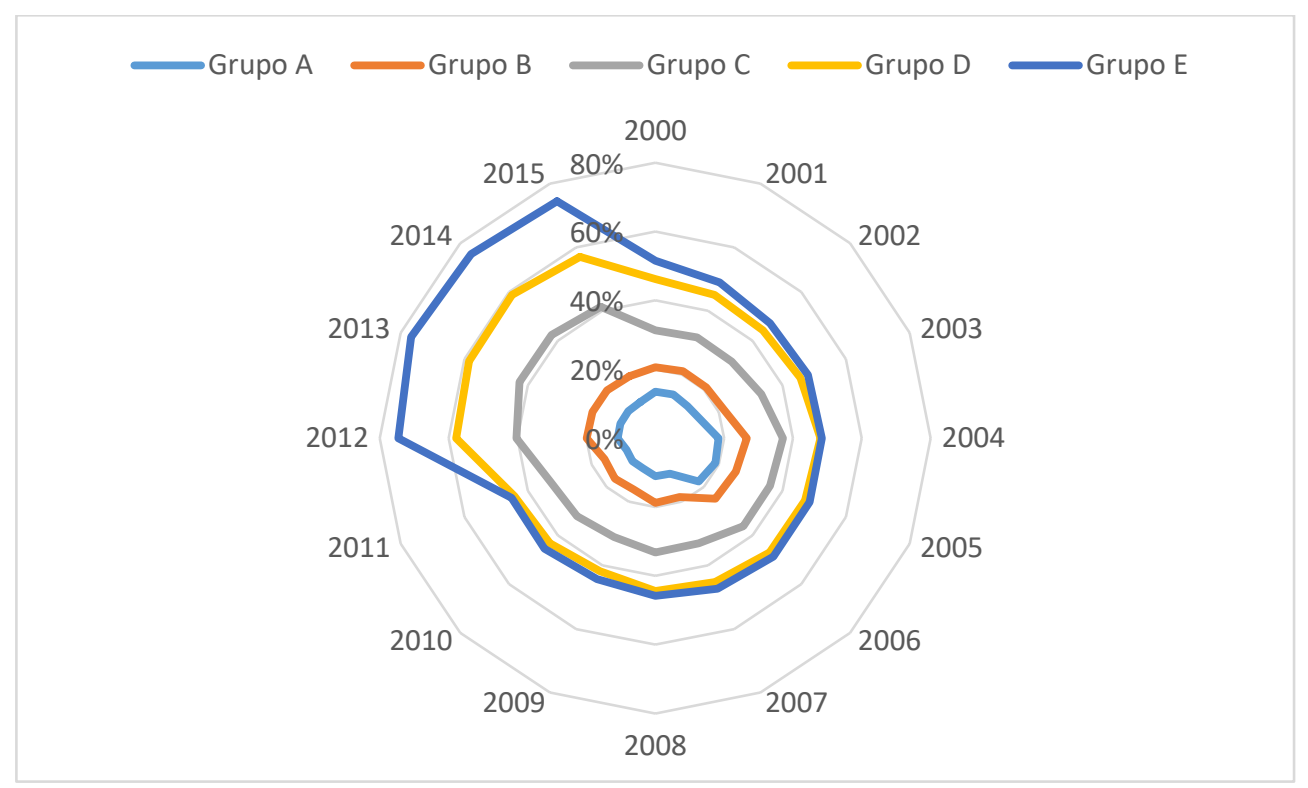

Fonte: Elab propia. 
Elección profesional y sesgo de selección: evaluación de los sistemas de admisión universitaria en Chile en un contexto de agenda pro-inclusión

Las tasas de selectividad (Gráfico 1) manifiestan un sesgo histórico que discrimina con base al perfil socioeconómico del alumnado, haciendo del contexto universitario un espacio escasamente inclusivo para sectores sociales de mayor vulnerabilidad. En efecto, las probabilidades de ser seleccionado mejoran conforme aumenta el estatus socioeconómico familiar y nivel educativo de los padres. Para el grupo de menor nivel socioeconómico (grupo A), de cada 10 postulantes tan solo 1 logra ser seleccionado en alguna de las vacantes ofrecidas. Baja tasa de inclusión, de comportamiento más o menos homogéneo dentro del período analizado, que en su fase temporal de mayor expansión (2004-2006) se posiciona en cifras que no superan el $19 \%$, alcanzando de media un $12 \%$ para igual período. Cifra que contrasta significativamente con los niveles de inclusión que alcanzan los postulantes procedentes de alto nivel socioeconómico (Grupo E), de los cuales 1 de cada 2 resulta ser seleccionado, incrementándose a partir del año 2012 a un $75 \%$. En consecuencia, en términos globales y con independencia relativa del año analizado, por cada 1 alumno proveniente de los sectores de menores ingresos que quedan seleccionados, alrededor de 5 de los estudiantes de altos ingresos logra igual objetivo. Así mismo, la gráfica informa que serían precisamente los grupos de mayores ingresos (Grupo E, D y C) quienes mejoran significativamente sus tasas de inclusión a partir del 2012, profundizando gradualmente las brechas respecto de los estudiantes más vulnerables.

Problemas de sesgo de selección que en todo caso y tal como se podrá evidenciar más adelante en el artículo, no son resultado exclusivo y directo de una fallida implementación de las medidas de una agenda pro inclusión, sino también se explican con ocasión de la incorporación de la oferta privada que antes de 2012 operaba al margen del SUA. A partir de dicha fecha se incorporan al Sistema Único de Admisión 8 universidades privadas, con una oferta educativa que al año 2014 bordeaba los 500 programas y representa el 35,4\% del total de postulantes seleccionados, los que pertenecen en mayor cuantía a los niveles socioeconómicos medios y altos. Si bien la incorporación de esta oferta privada antes autoexcluida del sistema de admisión hace más representativa las bases de datos y el estudio de los perfiles de quienes acceden a la educación superior en Chile, su incidencia debe ser necesariamente controlada al momento de estimar variaciones pre y post reforma al sistema de admisión a fin de evitar un sesgo de agregación.

Con la salvedad que supone para el análisis lo anteriormente expuesto, los datos informan que muy a pesar de los esfuerzos de dar equidad al sistema educativo mediante la estandarización del proceso en sus niveles intermedios: instauración de currículum nacional 
obligatorio, profesionalización de los docentes, sistemas de evaluación estandarizados; los problemas de segregación o inclusión diferenciada presentes en la educación secundaria se transmiten a la educación superior, limitan las posibilidades de interacción inter-clase y el intercambio/generación de capital simbólico y social de los estudiantes (BELLEI, 2013; OCDE, 2009; CANALES; DE LOS RÍOS, 2009). Sesgo de selección que se profundiza al contemplar las posibilidades de mantención y egreso de los estudiantes vulnerables en las instituciones universitarias fundamentalmente por dos razones. Primero, en comparación a los estudiantes de contextos socioeconómicos altos, para los estudiantes vulnerables el ingreso a la universidad supone una experiencia de ajuste demandante, ya que las capacidades desarrolladas en la educación secundaria se vuelven insuficientes para enfrentar los desafíos de la educación superior, aún más cuando se logra entrar a instituciones consideradas fuertemente selectivas académicamente (LEYTON; VÁSQUEZ; FUENZALIDA, 2012). Segundo, los estudiantes vulnerables históricamente han sido proclives a la deserción de estudios, principalmente por la falta de soportes familiares, sociales e institucionales que atenúen el impacto de eventos inesperados - pérdida del jefe de hogar, nacimiento de hijos- así como la inserción temprana en el mercado laboral, la que puede ocasionar la salida permanente de los estudiantes de sus carreras formativas (CANALES; DE LOS RÍOS, 2009). En especial, el riesgo de deserción que afecta a los alumnos de contextos vulnerables contribuye a la generación de inequidad y desequilibrio social, a la vez que desvirtúa los objetivos delegados a este nivel formativo (GONZÁLEZ; URIBE; GONZÁLEZ, 2005).

\section{Cuadro 1 - Concentración de los alumnos seleccionados en los procesos 2011}

y 2014, con ajustar y sin ajustar distribución (\%)

\begin{tabular}{|c|c|c|c|c|}
\hline \multirow[b]{2}{*}{ Categorías } & \multicolumn{2}{|c|}{ General } & \multicolumn{2}{|c|}{ Ajustada } \\
\hline & 2011 & 2014 & 2011 & 2014 \\
\hline $\begin{array}{l}\text { Dependencia } \\
\text { - Municipal } \\
\text { - Part Subvencionado } \\
\text { - Part Pagado }\end{array}$ & $\begin{array}{l}33,3 \\
50,7 \\
16,0 \\
\end{array}$ & $\begin{array}{l}25,7 \\
53,4 \\
20,9 \\
\end{array}$ & $\begin{array}{l}32,9 \\
50,7 \\
16,4 \\
\end{array}$ & $\begin{array}{l}28,7 \\
54,6 \\
16,7 \\
\end{array}$ \\
\hline $\begin{array}{l}\text { Primera Generación } \\
\text { - No } \\
\text { - Si }\end{array}$ & $\begin{array}{l}51,5 \\
48,5 \\
\end{array}$ & $\begin{array}{l}56,4 \\
43,6 \\
\end{array}$ & $\begin{array}{l}35,5 \\
64,5 \\
\end{array}$ & $\begin{array}{l}38,1 \\
61,9 \\
\end{array}$ \\
\hline $\begin{array}{ll} & \text { NSE } \\
- & \text { Bajo (A) } \\
- & \text { Medio Bajo (B) } \\
- & \text { Medio (C) } \\
- & \text { Medio alto (D) } \\
\text { - } & \text { Alto (E) } \\
\end{array}$ & $\begin{array}{r}6,3 \\
21,9 \\
31,8 \\
23,7 \\
16,3\end{array}$ & $\begin{array}{r}4,9 \\
18,1 \\
31,4 \\
24,6 \\
21,0\end{array}$ & $\begin{array}{r}6,2 \\
21,6 \\
31,6 \\
23,8 \\
16,7 \\
\end{array}$ & $\begin{array}{r}5,5 \\
19,9 \\
33,4 \\
24,4 \\
16,8 \\
\end{array}$ \\
\hline
\end{tabular}

Fuente: Elab propia. 
Elección profesional y sesgo de selección: evaluación de los sistemas de admisión universitaria en Chile en un contexto de agenda pro-inclusión

Nota: Concentración general contempla la distribución de la totalidad de la demanda que para los años respectivos resultó seleccionada en algún programa ofertado; la concentración ajustada, a fin de hacer las distribuciones comparables, contempla solo a los seleccionados de programas existentes en el proceso de admisión 2011, excluyendo por defecto a la oferta privada que se incorpora al SUA en 2012; Part=Particular; NSE= Nivel Socioeconómico.

El cuadro 1 informa el perfil del alumnado seleccionado en la oferta educativa disponible en los años 2011 y 2014 a fin de evidenciar los cambios suscitados con ocasión de las reformas al sistema de admisión. La distribución a nivel general y en referencia a la dependencia administrativa del colegio de procedencia constata, para el 2014, una pérdida significativa de presencia de alumnos de colegios municipales. Mientras en 2011 uno de cada tres estudiantes $(33,3 \%)$ que resultaban seleccionados en algún programa de pregrado provenía de un establecimiento educativo de carácter municipal, en la actualidad esta cifra es de tan solo un $25,7 \%$. La mayor alza en términos comparativos se registra en los alumnos de colegios particular pagados, quienes suben de un $16,0 \%$ a un $20,9 \%$. Tomando en cuenta que este conglomerado en términos poblacionales representa alrededor del $10 \%$ del estudiantado que egresa de la enseñanza media cada año, estaríamos en presencia de una sobre representación estadística de dicho cluster en el espacio universitario selectivo. Igualmente, al contemplar la totalidad de la demanda seleccionada se observan cambios en el perfil respecto de otras variables de clasificación. Se reduce la presencia de alumnos provenientes de familias cuyos padres no superan la enseñanza media como nivel educativo y buscan ser primera generación en acceder a la educación superior. Respecto del NSE se observa un aumento en la concentración en los grupos extremos de la distribución.

Cabe hacer presente que estos cambios y tendencias evidenciados son explicados principalmente con ocasión de la incorporación de los programas pertenecientes a las 8 universidades privadas que se integraron al SUA. De modo que al controlar el efecto de agregación a fin de hacer las distribuciones comparables, se constata una neutralización de la concentración de alumnos provenientes de colegios particulares pagados y de NSE Alto. El ajuste realizado pone en evidencia además la importancia que para efectos de universidades públicas y tradicionales tiene la participación de estudiantes primera generación, los cuales con independencia del año analizado representan alrededor del $63 \%$ de los postulantes seleccionados.

En consecuencia, si bien la generalidad de la oferta privada está fuertemente segmentada con base a atributos socioeducativos de su alumnado, en lo que a las universidades recientemente adscritas al SUA respecta, es posible afirmar que se componen en mayor medida por alumnos de mejor perfil socioeconómico, esto es, una mayor proporción 
de estudiantes provenientes de establecimientos particulares pagados, de altos ingresos y cuyos padres han alcanzado la educación superior.

Cuadro 2 - Diferenciales de concentración de alumnos seleccionados en procesos de admisión 2011 y 2014 según nivel de selectividad de los programas (\%)

\begin{tabular}{|l|r|r|r|r|r|r|r|r|r|}
\multicolumn{1}{c|}{} & \multicolumn{3}{c|}{ Baja } & \multicolumn{3}{c|}{ Media } & \multicolumn{3}{c|}{ Alta } \\
\hline \multicolumn{1}{c|}{ Categorías } & $\mathbf{2 0 1 4}$ & RC Gral & RC Ajust & $\mathbf{2 0 1 4}$ & RC Gral & RC Ajust & $\mathbf{2 0 1 4}$ & RC Gral & RC Ajust \\
\hline Dependencia & & & & & & & & \\
- Municipal & 31,3 & 0,72 & 0,86 & 25,3 & 0,74 & 0,85 & 15,5 & 0,81 & 0,89 \\
- Part Subvencionado & 59,9 & 1,14 & 1,10 & 55,0 & 0,99 & 1,06 & 34,0 & 0,95 & 1,05 \\
- Part Pagado & 8,8 & 2,40 & 1,24 & 19,8 & 1,89 & 1,15 & 50,5 & 1,12 & 1,01 \\
\hline Primera Generación & & & & & & & & & \\
- No & 27,9 & 1,44 & 1,22 & 41,0 & 1,32 & 1,13 & 68,3 & 1,07 & 1,03 \\
- Si & 72,1 & 0,89 & 0,95 & 59,0 & 0,86 & 0,94 & 31,7 & 0,88 & 0,95 \\
\hline NSE & & & & & & & & & 1,04 \\
- Bajo (A) & 7,6 & 0,65 & 0,77 & 4,4 & 0,74 & 0,91 & 0,7 & 1,21 \\
- Medio Bajo (B) & 26,9 & 0,77 & 0,87 & 16,6 & 0,74 & 0,88 & 5,0 & 0,92 & 1,07 \\
- Medio (C) & 35,7 & 1,08 & 1,12 & 32,8 & 0,92 & 1,02 & 17,9 & 0,91 & 1,03 \\
- Medio alto (D) & 21,9 & 1,30 & 1,15 & 26,2 & 1,04 & 1,04 & 24,7 & 0,89 & 0,96 \\
- Alto (E) & 7,9 & 2,40 & 1,21 & 20,0 & 1,91 & 1,14 & 51,7 & 1,11 & 1,00 \\
\hline
\end{tabular}

Fuente: Elab propia.

Nota: La selectividad del programa se calcula con base al comportamiento histórico que tendría la demanda (alumnos seleccionados) en razón del puntaje PSU, usando como año de referencia el proceso de admisión 2011. Alta $=$ al menos el 50\% del alumnado seleccionado sobre 650 puntos PSU, Media $=$ más del $50 \%$ del alumnado seleccionado con rangos de puntaje entre 550 y 650 puntos PSU, Baja=más del $50 \%$ de su alumnado seleccionado bajo 550 puntos PSU. Part=Particular; NSE= Nivel Socioeconómico; $\mathrm{RC}=$ es la razón de cocientes o riesgo relativo que establece el nivel de probabilidad de pertenecer a un determinado grupo en el 2014 respecto de la probabilidad registrada en 2011.

La configuración de la demanda está condicionada por los niveles de selectividad que tienen los programas, de modo que el perfil del alumnado puede variar al momento de controlar por el nivel de exigencia expresado en puntajes PSU (baja, media y alta). En efecto los datos informan de una mayor concentración de alumnos de colegios particulares pagados en los niveles de mayor selectividad. Dados sus históricos mejores niveles de logro en las pruebas estandarizadas (CONTRERAS; CORBALÁN; REDONDO, 2007; VALDIVIESO; ANTIVILO; BARRIOS, 2006), existiría una fuerte sobrerrepresentación de estos en las opciones universitarias altamente selectivas, es decir, en aquellos programas donde más del $50 \%$ del alumnado se caracteriza por alcanzar puntajes sobre los 650 puntos PSU. En el año 2014 el 50,5\% del alumnado seleccionado en programas de alta exigencia pertenecían a este 
Elección profesional y sesgo de selección: evaluación de los sistemas de admisión universitaria en Chile en un contexto de agenda pro-inclusión

conglomerado, participación que es un $12 \%$ superior a la existente en 2011, aunque dicha alza es fuertemente modelada al controlar el sesgo de agregación.

En los otros segmentos de selectividad (Media y Baja) la concentración de estudiantes de colegios particular pagados es claramente menos relevante y dejan de constituirse en una opción de preferencia, pese a que post-reforma esta presencia aumenta respecto de la evidenciada en 2011. Por su parte, los estudiantes de establecimientos públicos reducen su nivel de participación en todos los conglomerados de selectividad. En términos de concentración de este tipo de estudiantes al interior de cada cluster de selectividad se producen notorias reducciones respecto de 2011, las cuales son relevantes aun después de controlar el sesgo de agregación con ocasión de la incorporación de la oferta privada.

Del total de postulantes seleccionados en el año 2014, entre un 61,9\% y 43,6\%, según se ajuste o no la distribución, son alumnos que pretenden ser los primeros del grupo familiar en ingresar a la educación superior (Cuadro 1). Si bien esta importante cifra expresa en términos generales un mayor nivel de inclusión y democratización del espacio universitario, un análisis más detallado de las cifras evidencia una fuerte tendencia de segmentación en lo que a elección profesional respecta. A nivel general, el índice de concentración de alumnos primera generación al 2014 registra una reducción del 10\% respecto de la existente el año 2011, básicamente como resultado de la incorporación de un contingente de diferenciado perfil socioeducativo que accede a parte de la oferta privada incorporada al SUA. Esta reducción en la concentración de alumnos primera generación, aunque moderada por ajuste de sesgo, se replica en todos los niveles de selectividad analizados, estando las disminuciones más acentuadas en los niveles de alta selectividad (-12\%) y media selectividad (-14\%). Esta desigual concentración se explica por el sesgo cultural de las pruebas de admisión que dificulta a estos alumnos obtener altos puntajes PSU que posibiliten ingresar a programas selectivos. Se estima que una mayor valoración de la trayectoria escolar en detrimento de las pruebas de admisión reducirá este sesgo y permitirá una mayor inclusión a la diferenciada oferta educativa.

Igualmente, el análisis de concentración con base al atributo socioeconómico del alumnado seleccionado da cuenta de una mayor prevalencia de los niveles medios y altos, situación profundizada post reforma al sistema de admisión. A nivel general, los alumnos seleccionados pertenecientes a los conglomerados A y B han disminuido su participación en un 22,2\% y 17,3\% respectivamente (Cuadro 1). Concentración que además tiene un comportamiento diferencial con base al nivel de selectividad del programa elegido, haciendo de la presencia de los segmentos de menores ingresos en la oferta educativa de alta exigencia 
PSU un evento marginal. De cada cien postulantes seleccionados a un programa altamente selectivo, tan solo seis $(5,7 \%)$ pertenecen a los dos grupos de menores ingresos. Para los estudiantes provenientes de sectores socioeconómicos más vulnerables, una vez traspasado, no sin grandes dificultades, el escollo que involucra la PSU y la barrera de los 500 puntos, sus posibilidades educativas se limitan a decisiones profesionales vinculadas a programas de baja selectividad.

Si bien el sistema de educación superior, en especial el de carácter universitario, ha mejorado su tasa de cobertura haciéndose más inclusivo, su oferta educativa está fuertemente segmentada y condicionada por componentes de capital cultural y socioeconómico. Fragmentación que no solo es expresión de la dualización de la institucionalidad universitaria según adhieran o no al Sistema Único de Admisión, sino que se prolonga y profundiza en la oferta selectiva misma, haciendo de los programas de formación profesional de alta exigencia y eventualmente dotados de mayor calidad, una opción escasamente elegible para los segmentos sociales más vulnerables. Determinados espacios universitarios son profundamente homogéneos en cuanto a características socioculturales de su alumnado como expresión de una reproducción social determinista, que se manifiesta a pesar de la mayor movilidad social atribuida y de las eventuales mejoras que las reformas al sistema de admisión buscan instalar.

\section{Cuadro 3 - distribución de los alumnos seleccionados según nivel de exigencia-PSU del} programa y variables de segmentación, procesos 2011 y 2014

\begin{tabular}{|l|r|r|r|r|r|r|r|r|r|}
\multicolumn{1}{c|}{} & \multicolumn{3}{c|}{ Baja } & \multicolumn{3}{c|}{ Media } & \multicolumn{3}{c|}{ Alta } \\
\hline \multicolumn{1}{c|}{ Categorías } & $\mathbf{2 0 1 4}$ & $\mathbf{R C}$ Gral & RC Ajust & $\mathbf{2 0 1 4}$ & $\mathbf{R C}$ Gral & RC Ajust & $\mathbf{2 0 1 4}$ & RC Gral & RC Ajust \\
\hline Dependencia & & & & & & & & \\
- Municipal & 40,1 & 1,25 & 1,15 & $\mathbf{5 0 , 6}$ & $\mathbf{0 , 9 1}$ & 0,93 & 9,3 & 0,77 & 0,93 \\
- Part Subvencionado & 37,0 & 1,45 & $\mathbf{1 , 2 0}$ & $\mathbf{5 3 , 1}$ & 0,89 & 0,95 & 9,8 & 0,66 & 0,89 \\
- Part Pagado & $\mathbf{1 3 , 9}$ & $\mathbf{2 , 4 8}$ & $\mathbf{1 , 4 4}$ & $\mathbf{5 8 , 8}$ & 1,37 & 1,08 & 37,3 & 0,64 & 0,92 \\
\hline Primera Generación & & & & & & & & & \\
- No & 22,5 & 1,66 & 1,32 & 51,8 & 1,07 & 1,01 & 25,8 & 0,68 & 0,88 \\
- Si & 40,2 & 1,32 & 1,16 & 51,5 & 0,89 & 0,94 & 8,3 & 0,71 & 0,91 \\
\hline NSE & & & & & & & & & \\
- Bajo (A) & 50,6 & 1,11 & 1,01 & 47,0 & 0,90 & 0,98 & 2,4 & 0,99 & 1,25 \\
- Medio Bajo (B) & 48,1 & 1,25 & 1,11 & 47,5 & 0,85 & 0,92 & 4,3 & 0,83 & 1,07 \\
- Medio (C) & 36,9 & 1,46 & 1,25 & 54,2 & 0,88 & 0,93 & 8,9 & 0,68 & 0,90 \\
- Medio alto (D) & 29,01 & 1,69 & 1,31 & 55,2 & 0,95 & 0,97 & 15,8 & 0,64 & 0,87 \\
- Alto (E) & 12,2 & 2,50 & 1,41 & 49,2 & 1,41 & 1,09 & 38,6 & 0,64 & 0,92 \\
\hline
\end{tabular}

Fuente: Elab. propia. 
Elección profesional y sesgo de selección: evaluación de los sistemas de admisión universitaria en Chile en un contexto de agenda pro-inclusión

Nota: Part=Particular; la selectividad del programa se calcula con base al comportamiento histórico que tendría la demanda (alumnos seleccionados) en razón del puntaje PSU usando como año de referencia el proceso de admisión 2011. Alta $=$ al menos el 50\% del alumnado seleccionado sobre 650 puntos PSU, Media= más del 50\% del alumnado seleccionado con rangos de puntaje entre 550 y 650 puntos PSU, Baja=más del $50 \%$ de su alumnado seleccionado bajo 550 puntos PSU. Part=Particular; NSE= Nivel Socioeconómico; RC= es la razón de cocientes o riesgo relativo que establece el nivel de probabilidad de pertenecer a un determinado grupo en el 2014 respecto de la probabilidad registrada en 2011.

La problemática de inclusión educativa que registra el espacio universitario nacional se ilustra mediante el Cuadro 3. Esta tabla muestra la distribución de los estudiantes seleccionados en los diferentes programas en razón de atributos socioeducativos teóricamente relevantes. Obviando las lógicas diferencias que pudiesen observarse por institución y programa, a nivel general los datos informan que el contexto universitario de carácter selectivo es, pese a las reformas estructuradas, un espacio fuertemente discriminador que sobrerrepresenta a conglomerados de estudiantes de mejor capital cultural. Al 2014 (Cuadro 1), mientras siete de cada diez estudiantes de colegios particular pagados lograban ingresar a la oferta selectiva, tan solo un $23 \%$ de los provenientes de establecimientos municipales lo lograba. Probabilidad de ingresar que también son reducidas cuando los padres carecen de educación superior (23\%) y pertenecen al grupo socioeconómico Bajo (11\%) o Medio Bajo $(19,7 \%)$. En consecuencia se puede establecer, en lo que a oferta educativa universitaria selectiva se refiere, que esta continúa siendo privilegio, aunque no exclusivo, de alumnos de colegios pagados, de altos ingresos y con padres que han transitado previamente por igual experiencia. Distribución sesgada que se profundiza al momento de controlar con base al nivel de exigencia-PSU que exhibe el programa de la oferta que adhiere al SUA.

Con base a los niveles de selectividad del programa, los patrones de densidad acumulada en las distribuciones de los establecimientos públicos y subvencionados difieren significativamente respecto de los observados en quienes provienen de colegios particular pagados. En razón de este atributo de dependencia las distribuciones muestran patrones de acumulación inversos, evidenciando que los programas de baja selectividad son opción preferencial de estudiantes de colegios municipales (40\%) y particulares subvencionados (37\%). Por su parte, los alumnos de colegios particular pagados tienden a ser elegidos con mucha mayor frecuencia por parte de la oferta de mayor exigencia-PSU (37,3\%). Composición del sesgo de la distribución que, en el análisis de dependencia y selectividad, se ve modelado con posterioridad al 2011. 
El comportamiento distributivo anteriormente descrito se replica bajo la condición de alumno primera generación y proveniente de establecimientos que atiende a alumnado con bajos niveles de renta. En efecto, la opción educativa de estos segmentos (primera generación y grupos A y B) registran mayor densidad en su distribución en programas menos selectivos, manifestación de tendencia alcista entre los años analizados. El comportamiento de la variable NSE en los diferentes grupos de distribución es especialmente ilustrativo respecto de la segmentación socioeducativa que se prolonga en el contexto universitario, la cual hace mención a una marginal presencia de los niveles socioeconómicos bajo y medio bajo en programas de alta exigencia-PSU, la cual no supera el 2,4\% y 4,3\% respectivamente según datos 2014.

La segmentación del alumnado por atributos socioculturales se mantiene como una característica estructurante del sistema de educación universitaria, en especial conforme aumenta el nivel de exigencia-PSU del programa al que se postula. No obstante ello, los datos informan de cierta moderación al comparar las distribuciones de los procesos 2011-2014. Aunque puede ser prematuro establecerlo como tendencia, por precisar un mayor seguimiento y control de los datos, en lo inmediato se observa una relativa mayor mixturización en las distintas opciones profesionales. Así por ejemplo, se registra un crecimiento en las opciones educativas de baja y media selectividad por parte de los alumnos de colegios particular pagados y de altos ingresos. Distribución de probabilidad que es acrecentada post incorporación de las universidades privadas al SUA e incluso persiste, aunque atenuada, una vez realizado el ajuste por sesgo de agregación. Este mismo ajuste en la distribución da cuenta de un aumento en la probabilidad que tienen los estudiantes de menores ingresos de ser elegidos en programas de alta exigencia-PSU que, en el caso del grupo NSE A, alcanza a un $25 \%$ superior al registrado en 2011 ( $\mathrm{RC}$ ajustado=1,25).

Pese a que el déficit de capital cultural es un factor difícil de contrapesar al momento de rendir pruebas de selección universitaria, la participación de alumnos primera generación ha aumentado levemente. Situación similar presentan los estudiantes de los tres primeros NSE. Las pruebas de selección universitaria no tan solo se constituyen en un obstáculo difícil de superar para estos segmentos, sino que además profundiza la segmentación de las elecciones universitarias, haciendo de la presencia de estos grupos en las opciones profesionales de mayor prestigio social y rentabilidad económica un suceso esporádico y ocasional. Hacer del espacio universitario selectivo una opción inclusiva, más determinado por el talento y esfuerzo desplegado que por privilegios adscritos, continúa siendo tarea pendiente. Aunque se observa una mayor presencia de segmentos sociales más vulnerables en 
Elección profesional y sesgo de selección: evaluación de los sistemas de admisión universitaria en Chile en un contexto de agenda pro-inclusión

opciones académicas altamente selectivas, esto parece ser más resultado del azar y la casuística, presentando una escasa relevancia estadística como para hacer de ella tendencia. A pesar de ello, la revalorización de las trayectorias educativas alberga la posibilidad de un cambio en los procesos de reproducción de la estratificación socioeducativa instalada en la base del sistema educativo que resignifique la dedicación y la persistencia en la tarea.

Si bien reformas como esta tienen la posibilidad de democratizar el acceso a los espacios universitarios, queda como tarea pendiente igualar las posibilidades de permanencia entre los alumnos. La revalorización al esfuerzo desplegado durante la enseñanza secundaria será del todo insuficiente si esta formación no mejora en calidad y pertinencia y, por otra parte, las universidades no se hacen cargo de superar el déficit socioeducativo que traen los alumnos más vulnerables a fin de garantizar eficiencia educativa y éxito académico.

\section{Conclusiones}

La insatisfacción generalizada que provoca el sistema de selección universitario a nivel nacional ha llevado a los organismos responsables a implementar reformas para aplacar los efectos del determinismo social de variables estructurales como el nivel socioeconómico o el capital cultural en las posibilidades de ingreso a la educación superior; en resumidas cuentas, alejar del sistema universitario la imagen de reproductor de una estratificación social adscrita basada en credenciales y privilegios injustos.

Bajo esta intencionalidad pro-inclusión se creó la Prueba de Selección Universitaria y universalizó su aplicación, se reconfiguraron los factores de ponderación revalorizando la trayectoria y rendimiento escolar, a lo que se suma la reciente incorporación de una parte importante de la oferta privada, la cual adscribe a los mismos estándares y criterios del Sistema Único de Admisión. Siendo los períodos transcurridos breves como para identificar impactos de las reformas implementadas al interior del sistema, el análisis de los datos permite dar luces y perfilar tendencias respecto de las adscripciones profesionales que tienen y hacen los estudiantes que, una vez traspasada la barrera que supone el puntaje PSU, resultan ser seleccionados en algún programa de la oferta universitaria.

El análisis de los datos reporta que, a pesar de la expansión de la matrícula y el aumento en la participación de estudiantes que buscan hacerse de un cupo dentro de la oferta universitaria de carácter selectivo, perdura un sesgo histórico que discrimina con base a características económicas, sociales y culturales determinadas, haciendo del contexto 
universitario un espacio escasamente inclusivo para los sectores sociales de mayor vulnerabilidad.

Más allá del esfuerzo económico y académico que realizan estos alumnos para llegar a la educación superior, la barrera del puntaje PSU se constituye en un obstáculo difícil de sortear. Superado este sesgo de selección, se encuentran con una oferta educativa profundamente estratificada por atributos socioeconómicos. Existiría una sobrerrepresentación estadística en términos de distribución y concentración de estratos mejor dotados de capital económico y cultural. Aunque esta tendencia es profundizada con la incorporación de la oferta privada, su persistencia en el modelo ajustado informa de lo complejo que resulta para la política educativa actual horadar las barreras estructurales que condicionan el tipo de elección profesional. Elección que, para un importante segmento poblacional, es expresión más de un ajuste de expectativas que de un legítimo interés vocacional.

Programas e instituciones se distribuyen con base a un sesgo de homogeneidad interna (intra-grupo) y una heterogeneidad externa (entre-grupos). En consecuencia, aun cuando en general se mejoran los niveles de participación, se mantiene una distribución desigual, especialmente en los programas de alta selectividad asociados simbólicamente a status y rentas altas.

Si bien esta aseveración no es nueva en el campo de la investigación educativa superior, da cuenta de la lenta reestructuración del mecanismo de admisión que empujó a las universidades a reposicionarse en torno a cuáles son los indicadores válidos para una mayor inclusión sin sacrificar prestigio académico ni eficiencia educativa. La oferta de programas "inclusivos" se espera aumente a medida que crece el reconocimiento a la trayectoria escolar, apostando por indicadores alternativos como sería el otorgar mayor ponderación a las Notas de Enseñanza Media y el Ranking escolar. Se puede aportar a una mayor inclusión social en la educación superior sin alterar significativamente las tasas de aprobación, retención y titulación oportuna que exhiben instituciones y programas actualmente.

\section{Agradecimientos}

El equipo de investigadores del Centro de Investigación CIDCIE-UBB agradece al Departamento de Evaluación, Medición y Registro Educacional (DEMRE) por la oportuna disponibilidad de las bases de datos que permitieron elaborar este artículo. 
Elección profesional y sesgo de selección: evaluación de los sistemas de admisión universitaria en Chile en un contexto de agenda pro-inclusión

\section{Referencias}

BELLEI, Cristian. El estudio de la segregación socioeconómica y académica de la educación chilena. Estudios Pedagógicos, Valdivia, n. 39, p. 325-345, 2013.

BETTS, Julian; MOREL, Darlene. The determinants of undergraduate grade point average. The relative importance of family background high school recources, and peer group effects. Journal of Human Resources, Madison, v. 34, n. 2, p. 268-293,1999.

CANALES, Andrea; DE LOS RÍOS, Danae. Retención de estudiantes vulnerables en la educación universitaria. Revista Calidad en la Educación, Santiago, n. 30, p. 50-83, 2009.

CATALÁN, Ximena; SANTELICES, María. Rendimiento académico de estudiantes de distinto nivel socioeconómico en Universidades: el caso de la Pontificia Universidad Católica de Chile. Calidad en la Educación, Santiago, n. 40, p. 22-52, 2014.

CLIFFORDSON, Christina; ASKLING, Berit. Different grounds for admission: its effects on recruitment and achievement in medical education. Scandinavian Journal of Educational Research, London, n. 50, p. 45-62, 2006.

CONTRERAS, Dante; GALLEGOS, Sebastián; MENESES, Francisco. Determinantes del desempeño universitario: $;$ importa la habilidad relativa? Revista Calidad en la Educación, Santiago, n. 30, p. 17-48, 2009.

CONTRERAS, Marisol; CORBALÁN, Francisca; REDONDO, Jesús. Cuando la suerte está hechada: Estudio cuantitativo de los factores asociados al rendimiento en la PSU. Revista Electrónica Iberoamericana sobre Calidad, Eficacia y Cambio en Educación, Madrid, v. 5, n. 5, p. 259-263, 2007.

GEISER, Saul; STUDLEY, Roger. UC and the SAT: Predictive validity and differential impact of the SAT I and SAT II at the University of California. Educational Assessment, California, v. 8, n. 1, p. 1-26, 2002.

GIL, Francisco. Acceso a las universidades: una propuesta. Cuadernos del foro nacional de educación de calidad para todos. Chile: Santiago, 2006.

GIL, Francisco; PAREDES, Ricardo; SÁNCHEZ, Ignacio. El ranking de las notas: inclusión con excelencia. Centro de políticas públicas de la UC, Santiago, v. 8, n. 60, p. 3-19, 2013.

GONZÁLEZ, Luis; URIBE, Daniel; GONZÁLEZ, Soledad. Estudio sobre la repitencia y deserción en la educación superior chilena. Santigo: Instituto Internacional para la Educación Superior en América Latina y el Caribe, 2005.

LARROCAU, Tomás. Ranking de Notas Proceso de Admisión 2014. Santiago: CRUCH, 2014.

LEYTON, Daniel; VÁSQUEZ, Alba; FUENZALIDA, Valentina. La experiencia de estudiantes de contextos vulnerables en diferentes instituciones de educación superior universitaria (IESU): resultados de investigación. Calidad en la Educación, Santiago, n. 37, p. 61-97, 2012.

MEDINA, Alejandra; AGUIRRE, Mario; LUENGO, Luis. Predictibilidad de las notas de enseñanza media según establecimiento de origen sobre el rendimiento académico en estudiantes de Odontología. Educación Médica Superior, Havana, v. 28, n. 1, p. 65-73, 2014. 
MENESES, Francisco; BLANCO, Christian. La posición en el Rankig y el puntaje de ingreso en la PUC: implicancias prácticas. Santiago: Departamento de Estudio MINEDUC, 2006.

MENESES, Francisco; TORO, Javiera. Predicción de notas en derecho de la Universidad de Chile: ¿sirve el ranking? ISEES, Santiago, v. 10, p. 43-58, 2012.

MENESES, Francisco; PARRA, Álvaro; ZENTENO, Luis. Se puede mejorar el sistema de ingreso a las universidades chilenas? El uso del ranking en la Universidad Catolica de Chile, Universidad de Chile y Universidad de Santiago de Chile. Santiago: Universidad de Chile, 2005.

OCDE. Revisión de políticas nacionales de educación: la educación superior en Chile. Santiago: OCDE \& Banco Mundial, 2009.

PÉREZ, Cristhian; ORTIZ, Liliana; PARRA, Paula. Prueba de selección universitaria, rendimiento en enseñanza media y variables cognitivo-actitudianales en alumnos de Medicina. Revista de Educación, Ciencia y Salud, Concepción, v. 8, n. 2, p. 120-127, 2011. RODRÍGUEZ, Carlos; CASTILLO, Víctor. Calidad en la formación inicial docente: los déficits de las competencias pedagógicas y disciplinares en Chile. Revista Electrónica Actualidades Investigativas en Educación, San Pedro Montes de Oca, v. 14, n. 2, p. 1-25, 2014.

RODRÍGUEZ, Carlos; CASTILLO, Víctor. Stock profesional, eficiencia educativa y segmentación: análisis de los procesos de formación profesional en Chile. Civilizar: Ciencias Sociales y Humanas, Bogotá, v. 15, n. 29, p. 181-196, 2015.

RODRIGUEZ, Carlos; CASTILLO, Víctor; SAAVEDRA, Ricardo. Expectativa, cobertura y dominio curricular: percepciones del profesorado en la enseñanza de la matemática. Paradigma, Maracay Edo Aragua, v. 36, n. 2, p. 177-201, 2015.

SANZ, Rafael. Integración del estudiante en el sistema universitario: la turoría. Cuadernos de Integración Europea, Valencia, n. 2, p. 69-95, 2005.

VALDIVIESO, Pablo; ANTIVILO, Andrés; BARRIOS, Juan. Caracterización educacional y sociodemográfica de los estudiantes que rinden la PSU, postula y se matriculan en las universidades reunidas en el consejo de rectores. Calidad en la Educación, Santiago, n. 24, p. 319-361, 2006. 